\title{
AMENDMENTS
}

\section{Publisher Correction: Astrocytes mediate neurovascular signaling to capillary pericytes but not to arterioles}

Anusha Mishra, James P. Reynolds, Yang Chen, Alexander V. Gourine, Dmitri A. Rusakov and David Attwell

Correction to: Nature Neuroscience https://doi.org/10.1038/nn.4428, published online 26 October 2016.

In the version of this article initially published, in Fig. 1e,h the labels "D-ATP" should have read "D-AP5". The error has not been corrected in the original article.

Published online: 13 July 2020

https://doi.org/10.1038/s41593-020-0680-0

(C) The Author(s), under exclusive licence to Springer Nature America, Inc. 2020

\section{Publisher Correction: Rates, distribution and implications of postzygotic mosaic mutations in autism spectrum disorder}

Elaine T. Lim, Mohammed Uddin, Silvia De Rubeis, Yingleong Chan, Anne S. Kamumbu, Xiaochang Zhang, Alissa M. D'Gama, Sonia N. Kim, Robert Sean Hill, Arthur P. Goldberg, Christopher Poultney, Nancy J. Minshew, Itaru Kushima, Branko Aleksic, Norio Ozaki, Mara Parellada, Celso Arango, Maria J. Penzol, Angel Carracedo, Alexander Kolevzon, Christina M. Hultman, Lauren A. Weiss, Menachem Fromer, Andreas G. Chiocchetti, Christine M. Freitag, Autism Sequencing Consortium, George M. Church, Stephen W. Scherer, Joseph D. Buxbaum and Christopher A. Walsh

Correction to: Nature Neuroscience https://doi.org/10.1038/nn.4598, published online 17 July 2017.

In the version of this article initially published, in Fig. 2a, zeros were omitted from the $y$-axis labels on the histogram. They should read, from bottom to top: $0.01,0.02,0.03,0.04,0.05,0.06,0.07$. The error has not been corrected in the original article.

Published online: 14 July 2020

https://doi.org/10.1038/s41593-020-0681-z

(c) The Author(s), under exclusive licence to Springer Nature America, Inc. 2020 